\title{
THE CALCIUM DEFICIT DIET DOES NOT AFFECT BODY COMPOSITION, GLUCOSE, AND LIPID STATUS IN OVARIECTOMIZED RATS
}

\author{
Joanna Suliburska ${ }^{\bowtie 1}$, Iskandar Azmy Harahap ${ }^{1}$, Natalia Wawrzyniak ${ }^{1}$, \\ Anna Gramza-Michałowska² \\ ${ }^{1}$ Department of Human Nutrition and Dietetics, Poznań University of Life Sciences \\ Wojska Polskiego 31, 60-624, Poznań, Poland \\ 2Department of Gastronomy Sciences and Functional Foods, Poznań University of Life Sciences \\ Wojska Polskiego 31, 60-624 Poznań, Poland
}

\begin{abstract}
Background and objectives. Ovariectomy leads to an alteration of estrogen status associated with impaired calcium metabolism, reduced bone mineral density, and disorder in lipid metabolism. Calcium deficiency may enhance the effect of estrogen-deficiency condition. Therefore, this study aimed to define whether a calcium deficit in a diet affected the body composition, glucose, and lipid blood parameters in ovariectomized rats.

Materials and methods. This study included thirty female Wistar rats (three months old). The rats were randomly assigned into three groups: Group $1(n=10)$ consisted of rats in the control group (C) fed the standard diet, group $2(n=10)$ consisted of ovariectomized rats (OVX) fed the standard diet, and group $3(n=10)$ consisted of ovariectomized rats fed the calcium-deficit diet (OVXD). The body composition, blood glucose, and blood lipid profile were determined.

Results. Obtained results demonstrated that a calcium-deficient diet and a standard diet had a similar effect on feeding efficiency and body composition in ovariectomized rats. Moreover, the liver's relative weight, heart, kidneys, pancreas, brain, and femurs significantly decreased in both ovariectomized groups. The blood levels of cholesterol (CHOL), triglycerides (TG), low-density lipoprotein cholesterol (LDL-C), and high-density lipoprotein cholesterol (HDL-C) increased similarly in the ovariectomized groups OVX and OVXD in comparison to the control group. Glucose concentration was comparable between groups.

Conclusions. Calcium deficiency did not enhance the ovariectomy effect on body composition, glucose, and lipid status in rats during the 12-week study.
\end{abstract}

Keywords: body composition, glucose, lipid, ovariectomy, rats

\section{INTRODUCTION}

After menopause, decreased oestrogen levels alter orexigenic and anorexigenic factors leading to increased food intake and weight gain (Brown and Clegg, 2010). Additionally, due to the low concentration of estrogen

The National Science Centre granted the fund of study with the number 2018/29/B/NZ9/00461.

『joanna.suliburska@up.poznan.pl, https://orcid.org/0000-0002-0937-8427 
in the body, the lipid profile also changes and negatively affects postmenopausal women's health leading to increased total cholesterol, triglycerides, and low-density lipoprotein (LDL) cholesterol and decreased high-density lipoprotein (HDL) cholesterol in the blood serum (Tawfik et al., 2015). In postmenopausal women, lipolysis is reduced, while de novo lipogenesis in the liver increases, resulting in fat accumulation and fatty liver (Palmisano et al., 2017). Decreased HDL levels are most likely due to elevated liver lipase levels, while higher LDL levels may be due to these molecules' slow metabolism (Chamberlain et al., 2008).

Estrogen status is also associated with calcium metabolism. Estrogen deficiency alters intracellular calcium ions concentrations, which may impair lipid metabolism. In turn, calcium deficiency may cause energy expenditure, glucose, lipid, and bone metabolic dysregulation in a menopausal state (Park et al., 2020). It is suggested that low calcium in the diet may be an obesogenic factor and may increase the risk of insulin resistance development in early growth. Moreover, calcium inadequacy in early life may predispose to increase susceptibility to obesity and metabolic disorders later in life (Marotte et al., 2014). Some authors indicated the mechanism of calcium in the organism - the anti-obesity mechanism involved regulation of adipogenesis, modulation of fat metabolism and thermogenesis, and modification of gut microbiota (Zhang et al., 2019). Additionally, it was found that a low calcium diet increased cholesterol levels and an insufficient calcium status in early life predisposes to dyslipidemia and hepatic lipid accumulation (Alomaim et al., 2019; Li et al., 2018).

Several studies show that women after menopause usually intake a low calcium diet (Purdue-Smithe et al., 2017; Quesada-Gomez et al., 2013). It seems that calcium deficiency may enhance metabolic disorders and osteoporosis, often associated with menopause (Ko and Kim, 2020). Therefore, this study aimed to investigate the effect of a calcium deficit diet on the body composition, glucose, and lipid status in ovariectomized rats.

\section{MATERIALS AND METHODS}

\section{Animal grouping and study design}

Thirty female Wistar rats (three months old) were included in this experiment. The rats were purchased from the Wielkopolska Centre for Advanced Technologies of Adam Mickiewicz University (Poznań, Poland). The Local Ethics Committee approved the animal experiment in Poznan, Poland, registration number 34/2019. Subsequently, the animals were habituated by adapting to laboratory conditions during the first five days and housed individually in stainless steel cages coated with metal-free enamel and kept under cycles of 12 hours light to 12 hours dark. In the first stage of the experiment, twenty rats underwent ovariectomy. An expert on animal ovariectomy carried out the procedures. Ketamine and cepetor were used for anaesthesia during the surgery. After the surgery, the animals were fed a semi-synthetic diet based on AIN-93M (Reeves and Suppl, 1997) and drank distilled water ad libitum. After seven days, the rats were randomly assigned to three groups consisting of ten rats. Group 1 included rats in the control group (C), group 2 consisted of rats in the ovariectomized group (OVX) fed the standard diet, while group 3 included ovariectomized rats fed a calcium-deficit diet (OVXD). In the calcium-deficient diet, no calcium was added to the mineral mixture of AIN-93M. The intake of the diet was monitored daily. The animal study design is revealed in Figure 1 and the daily intake data are shown in Table 1. After 12 weeks of the experiment, all animals were euthanized by decapitation (after $12 \mathrm{~h}$ fasting), and blood samples were collected. Blood samples were collected in serumseparated tubes to obtain serum. The liver, kidney, heart, spleen, pancreas, and femur were dissected, weighed, and stored at $-80^{\circ} \mathrm{C}$. To determine animal suffering during the 12 weeks after surgery humane endpoint criteria were established as follows: sudden weight loss, inability to take food and water, vocalization, long-term diarrhea, apathy, chronic inflammation at the suturing site. After surgery, any of these symptoms were noted.

\section{Serum and tissues}

Each blood sample obtained in experimental studies was collected in serum separator tubes and left undisturbed at room temperature for $30 \mathrm{~min}$. Afterwards, these samples were centrifuged for $15 \mathrm{~min}$ at 2,500 rpm at $4^{\circ} \mathrm{C}$, and the supernatants were removed and stored at $-80^{\circ} \mathrm{C}$. The tissues (femur, liver, spleen, kidneys, 


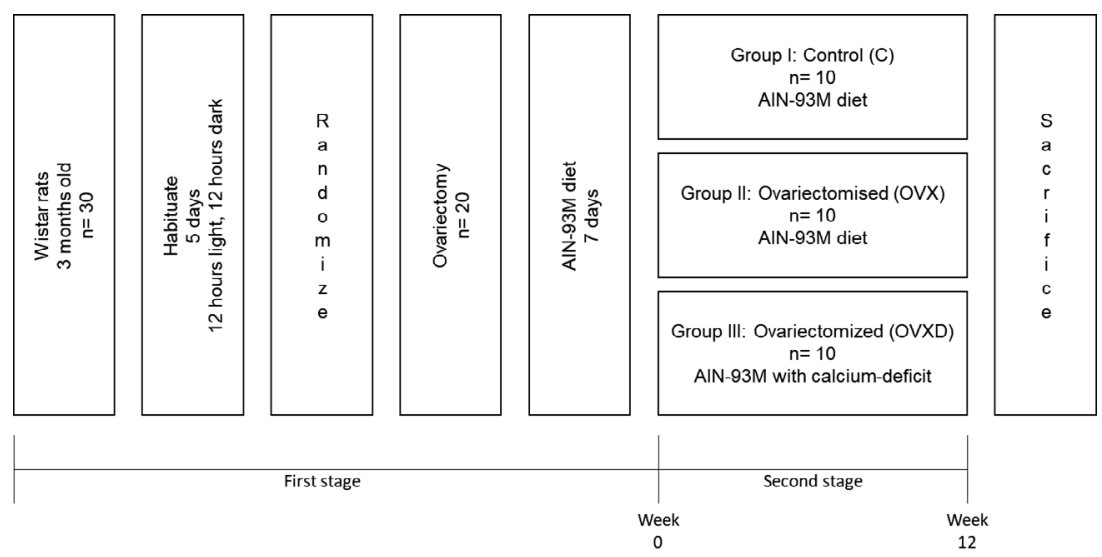

Fig. 1. Schematic of animal study design

heart, pancreas, brain) were dissected, weighed, and stored frozen at $-80^{\circ} \mathrm{C}$ for analysis.

Body composition and blood parameters analysis Body composition was examined by measuring body weight, body weight gain, fat tissue, free body liquid, and lean using Bruker's all-new 2nd generation minispec LF90II Body Composition Analyzer. At the same time, blood basal biochemical parameters were analyzed, including glucose (GLU), cholesterol (CHOL), triglycerides (TG), low-density lipoprotein cholesterol (LDL-C), and high-density lipoprotein cholesterol (HDL-C). Those serum concentrations were assayed using routine enzymatic methods in a certified diagnostic laboratory. Meanwhile, LDL was calculated with the Friedewald equation (Friedewald et al., 1972):

Cholesterol LDL, $\mathrm{mmol} / \mathrm{L}=$ $=\mathrm{TCH}, \mathrm{mmol} / \mathrm{L}-\mathrm{HDL}, \mathrm{mmol} / \mathrm{L}-\mathrm{TG}, \mathrm{mmol} / \mathrm{L} / 5$

\section{Statistical analysis}

Data were presented as means \pm standard deviation. The Shapiro-Wilk test was performed to check the normality of the distributions of the variables. Statistical significance was determined with a one-way analysis of variance (ANOVA) using Statistica (StatSoft, Tulsa, USA). When the ANOVA indicated a significant difference among the means, the differences were further evaluated using Tukey's test. The difference was considered significant when $p<0.05$.

\section{RESULTS}

\section{Result of body composition}

The results of the study are presented in Tables 1-3. We did not find a significant difference between group OVX and OVXD in any analyzed parameters.

Daily intake of the diet and water were comparable between the groups (Table 1). Body weight and body weight gain were significantly higher in groups OVX and OVXD than group C. Similarly, the body composition analysis showed differences between group $\mathrm{C}$ and groups OVX and OVXD. Fat tissue and free body liquid were markedly higher in ovariectomized rats (groups OVX and OVXD) than in healthy ones. It is worth mentioning that the values concerning fat tissue in ovariectomized groups were almost twice as highas in group C. In ovariectomized groups, the feeding efficiency was twice as high as in the control group and led to body weight gain.

When analyzing the relative weight of tissues, significant changes between the groups were observed (Table 2). The relative weight of the heart, kidneys, pancreas, brain, and femurs was significantly higher in group C than groups OVX and OVXD. On the other hand, the relative weight of the liver and pancreas differed statistically between group C and OVXD (it was lower in the group with calcium deficiency).

\section{Result of glucose and lipid parameters}

The analysis of biochemical parameters in serum showed that the rats' lipid profiles from the control 
Suliburska, J., Harahap, I. A., Wawrzyniak, N., Gramza-Michałowska, A. (2021). The calcium deficit diet does not affect body composition, glucose, and lipid status in ovariectomized rats. Acta Sci. Pol. Technol. Aliment., 20(4), 459-464. http://dx.doi.org/ 10.17306/J.AFS.2021.1004

Table 1. The profiles of diet intake, body composition at the end of the study, and feed efficacy

\begin{tabular}{lccc}
\hline \multirow{2}{*}{ Profiles } & \multicolumn{3}{c}{ Group } \\
\cline { 2 - 4 } & C & OVX & OVXD \\
\hline Daily food intake, g & 25.08 & 25.11 & 26.52 \\
& \pm 0.63 & \pm 1.70 & \pm 2.18 \\
Daily water intake, ml & 49.91 & 44.65 & 40.39 \\
& \pm 5.30 & \pm 5.10 & \pm 6.86 \\
Body weight, g & 325.86 & 421.90 & 441.90 \\
& $\pm 25.97^{\mathrm{a}}$ & $\pm 55.10^{\mathrm{b}}$ & $\pm 70.97^{\mathrm{b}}$ \\
Body weight gain, g & 54.96 & 106.20 & 120.18 \\
& $\pm 17.99^{\mathrm{a}}$ & $\pm 36.08^{\mathrm{b}}$ & $\pm 47.73^{\mathrm{b}}$ \\
Fat tissue, g & 113.66 & 225.68 & 246.19 \\
& $\pm 42.99^{\mathrm{a}}$ & $\pm 66.19^{\mathrm{b}}$ & $\pm 83.14^{\mathrm{b}}$ \\
Free body liquid, g & 34.08 & 52.90 & 55.98 \\
& $\pm 7.02^{\mathrm{a}}$ & $\pm 12.33^{\mathrm{b}}$ & $\pm 15.99^{\mathrm{b}}$ \\
Lean, g & 194.64 & 199.76 & 194.82 \\
& \pm 21.21 & \pm 29.27 & \pm 24.77 \\
Feed efficacy & 2.60 & 5.00 & 5.32 \\
& $\pm 0.82^{\mathrm{a}}$ & $\pm 1.66^{\mathrm{b}}$ & $\pm 1.88^{\mathrm{b}}$ \\
\hline
\end{tabular}

C - the control group, OVX - the ovariectomized group, OVXD - the ovariectomized group with calcium-deficit diet.

a,bSignificant differences between groups: ANOVA and Tukey's test.

Table 2. The relative weight of tissues in rats, $\%$ body weight

\begin{tabular}{lccc}
\hline \multirow{2}{*}{ Tissues } & \multicolumn{3}{c}{ Group } \\
\cline { 2 - 4 } & \multicolumn{1}{c}{ C } & OVX & OVXD \\
\hline Liver & $2.82 \pm 0.24^{\mathrm{b}}$ & $2.49 \pm 0.68^{\mathrm{ab}}$ & $2.21 \pm 0.17^{\mathrm{a}}$ \\
Heart & $0.34 \pm 0.03^{\mathrm{b}}$ & $0.26 \pm 0.03^{\mathrm{a}}$ & $0.25 \pm 0.02^{\mathrm{a}}$ \\
Kidneys & $0.65 \pm 0.07^{\mathrm{b}}$ & $0.47 \pm 0.05^{\mathrm{a}}$ & $0.47 \pm 0.05^{\mathrm{a}}$ \\
Spleen & $0.19 \pm 0.03$ & $0.17 \pm 0.02$ & $0.17 \pm 0.02$ \\
Pancreas & $0.33 \pm 0.06^{\mathrm{b}}$ & $0.28 \pm 0.05^{\mathrm{ab}}$ & $0.27 \pm 0.04^{\mathrm{a}}$ \\
Brain & $0.59 \pm 0.04^{\mathrm{b}}$ & $0.47 \pm 0.07^{\mathrm{a}}$ & $0.47 \pm 0.08^{\mathrm{a}}$ \\
Femures & $0.66 \pm 0.06^{\mathrm{b}}$ & $0.52 \pm 0.06^{\mathrm{a}}$ & $0.48 \pm 0.07^{\mathrm{a}}$ \\
\hline
\end{tabular}

Data are presented as mean \pm standard deviation.

$\mathrm{C}$ - the control group, OVX - the ovariectomized group, OVXD - the ovariectomized group with calcium-deficit diet. ${ }^{\mathrm{a}, \mathrm{b}}$ Significant differences between groups: ANOVA and Tukey's test.
Table 3. Blood basal biochemical parameters in rats, $\mathrm{mg} / \mathrm{dl}$

\begin{tabular}{lccc}
\hline \multirow{2}{*}{ Parameters } & \multicolumn{3}{c}{ Group } \\
\cline { 2 - 4 } & C & OVX & OVXD \\
\hline GLU & $109.60 \pm 14.11$ & $103.80 \pm 11.72$ & $110.55 \pm 12.13$ \\
CHOL & $92.10 \pm 14.25^{\mathrm{a}}$ & $148.58 \pm 33.44^{\mathrm{b}}$ & $143.34 \pm 28.56^{\mathrm{b}}$ \\
TG & $77.37 \pm 10.88^{\mathrm{a}}$ & $115.61 \pm 40.56^{\mathrm{b}}$ & $115.06 \pm 39.80^{\mathrm{b}}$ \\
LDL-C & $8.24 \pm 2.72^{\mathrm{a}}$ & $23.79 \pm 6.35^{\mathrm{b}}$ & $25.64 \pm 7.52^{\mathrm{b}}$ \\
HDL-C & $85.03 \pm 11.23^{\mathrm{a}}$ & $124.31 \pm 24.53^{\mathrm{b}}$ & $119.10 \pm 20.02^{\mathrm{b}}$ \\
\hline
\end{tabular}

Data are presented as mean \pm standard deviation.

$\mathrm{C}$ - the control group, OVX - the ovariectomized group, OVXD - the ovariectomized group with calcium deficit diet, GLU - glucose, CHOL - cholesterol, TG - triglycerides, LDL-C - low-density lipoprotein cholesterol, HDL-C - high-density lipoprotein cholesterol.

a,bignificant differences between groups: ANOVA and Tukey's test.

group differed significantly from that of ovariectomized rats (Table 3). Total cholesterol, LDL-C, HDL-C, and triglyceride levels were higher in the ovariectomized groups (OVX and OVXD) than group C. The LDL-C concentration attracted the most attention, as it is three times higher in ovariectomized groups than in the control group. Glucose concentration was comparable between groups.

\section{DISCUSSION}

The most important finding in this study is that low calcium with a standard diet does not affect body mass and lipid and glucose status in ovariectomized rats. However, several studies show the connection between calcium intake, lipid metabolism, and body composition (Hsu and Culley, 2006; Park et al., 2020). The lack of confirmation with the results of other studies may be because we used a standard diet in this experiment. Using an atherogenic diet or high lipid diet resulted in significant lipid disorders in experimental animals with calcium deficit (Hsu and Culley, 2006; Park et al., 2020). It was found that calcium deficiency changed the expression of genes related to lipid metabolism in the liver, calcium level in the diet influenced on fat absorption and fecal fat extraction, and low calcium intake generated lipid peroxidation (Li et al., 2018; Marotte 
et al., 2014; Zhang et al., 2019). Additionally, Park et al. (2020) showed that ovariectomy-induced obesity in rats and a high-fat diet exacerbated menopausal symptoms, including increased body fat mass. It seems that calcium deficiency may exert the detrimental effect of high cholesterol and fat level in the diet on lipid metabolism and fat accumulation in tissues. In our study, the effect of low estradiol caused by ovariectomy may be large enough for body composition and lipid metabolism that the lack of any additional harmful factor (e.g., high fat diet) suppresses the effects of a low calcium supply in the organism of ovariectomized rats.

Paradis and Cabanac (2005) found that different calcium levels did not affect body weight in healthy rats on the standard diet. In this experiment mentioned, the authors explained that calcium deprivation had some metabolic influence, and the defense mechanism adjusts body size to calcium availability.

There are several limitations in this study that may have affected its results. No sham group was included in the study, and therefore no effect of sham surgery on rats was noted. Moreover, we did not include parameters such as serum calcium level, serum parathyroid hormone, serum creatinine, serum estradiol, and bone mineral density which may broaden interpretation and discussion of the results.

\section{CONCLUSIONS}

This study can be summarised by stating that calcium deficiency does not alter the ovariectomy effect on anthropometry and biochemical parameters in rats during the 12-week study.

\section{REFERENCES}

Alomaim, H., Griffin, P., Swist, E., Plouffe, L. J., Vandeloo, M., Demonty, I., ..., Bertinato, J. (2019). Dietary calcium affects body composition and lipid metabolism in rats. PLoS ONE, 14(1), 1-21. https://doi.org/10.1371/ journal.pone.0210760

Brown, L. M., Clegg, D. J. (2010). Central effects of estradiol in the regulation of food intake, body weight, and adiposity. J. Steroid Biochem. Molec. Biol., 122(1-3), 65-73. https://doi.org/10.1016/j.jsbmb.2009.12.005

Chamberlain, A. M., Folsom, A., Schreiner, P. J., Eric Boerwinkle, Ballantyne C. M. (2008). Low-density lipoprotein and high-density lipoprotein cholesterol levels in relation to genetic polymorphisms and menopausal status: The atherosclerosis risk in communities (ARIC) study. Atherosclerosis, 200(2), 322-328. https:// doi.org/10.1016/j.atherosclerosis.2007.12.045

Friedewald, W. T., Levy, R. I., Fredrickson, D. S. (1972). Estimation of the concentration of low-density lipoprotein cholesterol in plasma, without use of the preparative ultracentrifuge. Clin. Chem., 18(6), 499-502.

Hsu, H. H. T., Culley, N. C. (2006). Effects of dietary calcium on atherosclerosis, aortic calcification, and icterus in rabbits fed a supplemental cholesterol diet. Lipids Health Diseas., 5, 16. https://doi.org/10.1186/1476511X-5-16

Ko, S. H., Kim, H. S. (2020). Menopause-associated lipid metabolic disorders and foods beneficial for postmenopausal women. Nutrients, 12, 1. https://doi.org/10.3390/ nu12010202

Li, P., Chang, X., Fan, X., Fan, C., Tang, T., Wang, R., Qi, K. (2018). Dietary calcium status during maternal pregnancy and lactation affects lipid metabolism in mouse offspring. Sci. Rep., 8(1), 1-10. https://doi.org/10.1038/ s41598-018-34520-6

Marotte, C., Bryk, G., Gonzales Chaves, M. M. S., Lifshitz, F., Pita Martín De Portela, M. L., Zeni, S. N. (2014). Low dietary calcium and obesity: A comparative study in genetically obese and normal rats during early growth. Eur. J. Nutr., 53(3), 769-778. https://doi.org/10.1007/ s00394-013-0581-z

Palmisano, B. T., Zhu, L., Stafford, J. M. (2017). Estrogens in the regulation of liver lipid metabolism. Adv. Exp. Med. Biol., 1043, 227-256. https://doi.org/10.1007/9783-319-70178-3

Paradis, S., Cabanac, M. (2005). Calcium deficiency cannot induce obesity in rats. Physiol. Behav., 85(3), 259-264. https://doi.org/10.1016/j.physbeh.2005.03.026

Park, S., Kang, S., Kim, D. S. (2020). Severe calcium deficiency increased visceral fat accumulation, down-regulating genes associated with fat oxidation, and increased insulin resistance while elevating serum parathyroid hormone in estrogen-deficient rats. Nutr. Res., 73, 4857. https://doi.org/10.1016/j.nutres.2019.09.008

Purdue-Smithe, A. C., Whitcomb, B. W., Szegda, K. L., Boutot, M. E., Manson, J. E., Hankinson, S. E., ..., Bertone-Johnson, E. R. (2017). Vitamin D and calcium intake and risk of early menopause. Am. J. Clin. Nutr., 105, 6, 1493-501. https://doi.org/10.3945/ajcn.116.145607

Quesada-Gomez, J. M., Diaz-Curiel, M., Sosa-Henriquez, M., Malouf-Sierra, J., Nogues-Solan, X., Gomez-Alonso, C., ..., Delgadillo, J. (2013). Low calcium intake 
Suliburska, J., Harahap, I. A., Wawrzyniak, N., Gramza-Michałowska, A. (2021). The calcium deficit diet does not affect body composition, glucose, and lipid status in ovariectomized rats. Acta Sci. Pol. Technol. Aliment., 20(4), 459-464. http://dx.doi.org/ 10.17306/J.AFS.2021.1004

and inadequate vitamin D status in postmenopausal osteoporotic women. J. Steroid Biochem. Mol. Biol., 136, 175-177. https://doi.org/10.1016/j.jsbmb.2012.10.013

Reeves, P. G., Suppl, M. (1997). Components of the AIN-93 diets as improvements in the AIN-76A diet. J. Nutr., 127, 5, 838-841. https://doi.org/10.1093/jn/127.5.838S

Tawfik, S. H., Mahmoud, B. F., Saad, M. I., Shehata, M., Kamel, M. A., Helmy, M. H. (2015). Similar and additive effects of ovariectomy and diabetes on insulin resistance and lipid metabolism. Biochem. Res. Int., 2015.ID 567945. https://doi.org/10.1155/2015/567945

Zhang, F., Ye, J., Zhu, X., Wang, L., Gao, P., Shu, G., ..., Wang, S. (2019). Anti-obesity effects of dietary calcium: The evidence and possible mechanisms. Int. J. Mol. Sci., 20(12), 3072. https://doi.org/10.3390/ijms20123072 